\title{
Antioxidant therapy to improve or resolve atherosclerosis; new hopes and current trends
}

\author{
Banafsheh Yalameha* ${ }^{(\mathbb{D}}$ \\ Department of Biochemistry, School of Medicine, Lorestan University of Medical Sciences, Khorramabad, Iran
}

\section{A R T I C L E I N F 0}

Article Type:

Mini-Review

\section{Article History:}

Received: 5 May 2018

Accepted: 9 January 2019

ePublished: 28 February 2019

\section{Keywords:}

Atherosclerosis, Antioxidant,

Cardiovascular disease, Coronary

artery disease, Oxidative stress,

Insulin resistance

\begin{abstract}
A B S T RAC T
Atherosclerosis is a chronic inflammatory disease occurring in arterial wall over many decades, often leading to acute cardiovascular event. The different mechanisms are involved in the development of this disease. Oxidative stress process is recognized as an important factor for initiation and progression of atherosclerosis. Many therapeutic approaches exist for controlling, preventing, and treating the disease. Antioxidants are the main compounds that inhibit oxidative stress by by removing free radicals and including protective effect against cardiovascular disease like atherosclerosis. Recently, several drugs about novel perspectives have been discovered which can improve lipid profiles. This review outlines the anti-atherosclerotic property of antioxidants in prevention and improvement atherosclerosis and its new hopes.
\end{abstract}

Implication for health policy/practice/research/medical education:

Antioxidant therapy can be useful in improving and treatment of atherosclerosis while further research on the issue is still required to extend our knowledge.

Please cite this paper as: Yalameha B. Antioxidant therapy to improve or resolve atherosclerosis; new hopes and current trends. J Nephropharmacol. 2019;8(2):e18. DOI: 10.15171/npj.2019.18

An introduction to atherosclerosis

Coronary artery disease (CAD) is known major leading causes of death in developed countries over recent decades. It has been estimated that 15.5 million adults (6.2\% of the adult population) are involved in CAD in the United States (1). Atherosclerosis is a chronic inflammatory vascular disease in arterial intima and established the major leading causes of death in the United States and Europe (2). The incidence of atherosclerosis is correlated with disorder of several biological pathways; such as angiogenesis, inflammatory response and immune response, adipogenesis, lipid metabolism, oxidative stress, insulin resistance and diabetes (3).

In 1995, it was suggested that selective retention of lipoprotein in the artery wall is the initiating step in atherogenesis. The disease is characterized by the presence of atherosclerotic plaques consisting of lipids, immune cells, and debris in the arterial intima. Plaques develop at predisposed regions characterized by disturbed blood flow dynamics, such as curvature and branch points (4). Several risk factors can be the cause of atherosclerotic, such as diabetes mellitus, hypertension, hypercholesterolemia, obesity, age, sex, inadequate lifestyle, and cigarette smoking (5).

\section{Role of oxidative stress in atherosclerosis}

Imbalance between the generation of reactive oxygen species (ROS) and the antioxidant causes oxidative stress. The different signals such as UV light, chemicals, cigarette smoke, microbial toxins stimulate oxidative stress process in body cells. This process is related to the pathogenesis of different cardiovascular diseases such as atherosclerosis through different mechanisms (6).

Disorders of lipid metabolism increase the level of blood low-density lipoprotein cholesterol (LDL-C) and its aggregation in vascular wall. Endothelial cells, 
smooth muscle cells (SMCs), and macrophages as the sources of ROS, which oxidize LDL-C to oxidized LDL (ox-LDL). These oxidation reactions of lipid induce inflammation processes and lead to the production of inflammatory cytokines. Macrophages attract Ox-LDL and convert to foam cells. Apoptosis and growth factors released from foam cells, induce SMCs proliferation and migration into the intima. Finally, a fibrous plaque formed and rupture of fibrous plaque leads to thrombus development and occlusion of the vessel $(5,7)$. Generally, the oxLDLs involved in several biological pathways including enhancing the expression of growth factors and related receptors, inducing ECM modification, activation phospholipase $\mathrm{D}$, lactosylceramide generation and the sphingomyelin/ceramide (8). Additionally, another mechanism of oxidative stress (by $\mathrm{H} 2 \mathrm{O} 2$ ) that induces atherogenesis is through the activation of transcription factors such as nuclear factor-kappa $\mathrm{B}(\mathrm{NF}-\mathrm{\kappa} \mathrm{B})$ and activator protein 1 (AP-1). These factors interfere with the expression of adhesion molecules such as intercellular adhesion molecule-1 (ICAM-1), vascular cell adhesion molecule-1 (VCAM-1), E-selectin and other chemokines and cytokines. All these agents cause activation of NF- $\kappa \mathrm{B}$ pathway that can be inactivated by antioxidants and antiinflammatory agents $(9,10)$.

Different enzymatic agents cause modification of LDL-C by oxidative stress that can refer to inducible nitric oxide synthase, NADPH oxidase, lipoxygenase, xanthine oxidase, myeloperoxidase, hemoxygenase-1, and ceruloplasmin (11). To reduce the oxidative stress conditions, the administration of antioxidants can be effective, in which a wide range of research has been shown. The present review outlines the role of antioxidant in improvement and treatment atherosclerosis and focusing on new hopes in atherosclerosis.

\section{Materials and Methods}

PubMed/Medline, EBSCO, Embase, Web of Science, directory of open access journals (DOAJ), Scopus, and Google Scholar was searched (2000 to 2017) using the following keywords: atherosclerosis, antioxidant, cardiovascular disease, coronary artery disease, oxidative stress, insulin resistance, reactive oxygen species, diabetes mellitus, hypertension, hypercholesterolemia, macrophage, intracellular adhesion molecules and vascular cell adhesion molecule.

\section{Results}

Antioxidant therapy in atherosclerosis

Antioxidants are widely considered to be the most important of compounds in prevention or treatment of different diseases (e.g., cancer, Alzheimer's disease, arthritis, and diabetes) (12). Numerous studies have confirmed the role of antioxidants in amelioration and the treatment of cardiovascular diseases such as atherosclerosis. For example, vitamin C, vitamin E, and polyphenols seem to be effective for cardiovascular diseases in clinical trials (13). The enzymatic (e.g., glutathione peroxidase, superoxide dismutases, and catalase) and non-enzymatic systems to be used against ROS which can prevent cell membrane oxidation (14).

The types of non-enzymatic antioxidants are included metabolic antioxidants and nutrient antioxidants. Metabolic antioxidants as endogenous antioxidants produced in the body that refers to glutathione, L-ariginine, bilirubin, coenzyme Q10, uric acid, melatonin, metalchelating proteins, lipoic acid, and transferrin. However, nutrient antioxidants exist in foods or supplements such as vitamin E, vitamin C, carotenoids, omega-3 and omega- 6 fatty acids, selenium, and flavonoids (15). For example, it has been suggested that uric acid increases cytokine production and inflammatory responses and additionally, the atherosclerotic lesion can be inhibited by lipoic acid supplement and unconjugated bilirubin in mice and human respectively. Vitamin E supplement possesses beneficial effect in the atherosclerotic lesion and delays its progression (8). A recent study illustrated that tocopherols decrease SMC proliferation, platelet aggregation, and lipid peroxidation (16). The anti-atherosclerotic effect of vitamin $\mathrm{C}$ is through inhibition of apoptosis and proapoptotic activity, induction of collagen synthesis, enhancing endothelial nitric oxide synthase (12).

Recent review literature on this topic found that vitamin D can inhibit ROS-generating enzyme NADPH oxidase activity and inflammatory mediators in oxidative vascular injuries (17). Flavonoids as herbal antioxidants have different therapeutic activities and neutralize free radicals. The antioxidant effects of these compounds in atherosclerosis are via preventing LDL oxidation in macrophage and reducing Ox-LDL attraction by macrophage receptors (18).

\section{Current trends of atherosclerosis}

Recently, many new strategies have been used to prevent or treat atherosclerosis. Here we will refer to some of these strategies. Inflammatory processes play an important role in the pathogenesis of atherosclerosis that can be used as therapeutic targets. The study by Khan et al showed several inflammatory pathways appeared in animal models, which can be targeted by new anti-atherosclerotic therapeutics including inhibition of interleukin-1 $\beta$ (IL-1 $\beta$ ), P selectin, serine protease and 5-lipoxygenase. The effect of IL- $1 \beta$ in atherosclerosis is through regulating leukocyte adhesion molecules, proliferation of VSMCs and production of other cytokines. Administration of IL-1 $\beta$ blockade can improve endothelial regrowth and cardiac remodeling and decrease cardiomyocyte apoptosis (19).

RVX-208 as BET bromodomain inhibitor is well known 
in prevention and treatment of atherosclerosis or other metabolic disorders. This new drug can increase level of apolipoprotein A-I (apoA-I), high-density lipoprotein (HDL), pre- $\beta-\mathrm{HDL}$, and $\alpha-1 \mathrm{HDL}$. The mechanism of RVX-208 in apoA-I level regulation is by binding to the BET protein and increasing apoA-I gene transcription that results in the production of this protein (20). Proprotein convertase subtilisin/kexin type 9 (PCSK9) is secretory serine protease that can increase circulating LDL-cholesterol by decreased liver LDL receptor. PCSK9 inhibitors as novel pharmacological treatment have shown that decrease circulating LDL-C via blocking PCSK9-mediated degradation of the LDL receptor (21). Recent evidence suggests that lymphangiogenic therapies can prevent the development of atherosclerosis through lipoprotein transport and delaying atherosclerotic plaque formation (22). More recently, it was demonstrated that. natural antioxidants in herbal medicines such as, Rhus coriaria, Anethum graveolens, Berberis integerrima Bunge, Glycyrrhiza glabra, Artemisia spp, Vaccinium myrtillus L, Crocus Sativus L, Gundelia tournefortii L, Hypericum perforatum, Curcuma longa, Malus domestica, Nigella sativa L, Silybum marianum, Trifolium pratense L, and Portulaca oleracea L can help to prevent the free radical damage associated with the development of atherosclerosis (23).

\section{Conclusion}

Atherosclerosis is a complex multifocal arterial disease associated with large and moderate arteries. It is caused by the interaction of multiple genetic and environmental factors. Oxidative stress plays a vital role in the pathogenesis of atherosclerosis and the use of antioxidants can prevent the progression of this disease. We conclude that antioxidant therapy can be useful in improving and treatment of atherosclerosis while further research on the issue is still required to extend our knowledge.

\section{Authors' contribution}

BY is the single author of the paper.

\section{Conflicts of interest}

The author declared no competing interests.

\section{Ethical considerations}

Ethical issues (including plagiarism, data fabrication, double publication) have been completely observed by the author.

\section{Funding/Support}

None.

\section{References}

1. Wilson PWFE, O’Donnell CJ. Epidemiology of Chronic Coronary Artery Disease. In: de Lemos J, Omland T, eds. Chronic Coronary Artery Disease: A Companion to Braunwald's Heart Disease E-Book. Elsevier Health Sciences; 2017.

2. Tyroler HA. Coronary heart disease epidemiology in the 21st century. Epidemiol Rev. 2000;22:7-13.

3. Chen WJ, Yin K, Zhao GJ, Fu YC, Tang CK. The magic and mystery of microRNA-27 in atherosclerosis. Atherosclerosis. 2012;222(2):314-23. doi: 10.1016/j. atherosclerosis.2012.01.020.

4. Monaco C, Lutgens E. Atherosclerosis- a short history. In: Krams R, Bäck M, eds. ESC Textbook of Vascular Biology. United States: Oxford University Press; 2017.

5. Li H, Horke S. Förstermann U. Vascular oxidative stress, nitric oxide and atherosclerosis. Atherosclerosis. 2014; 237:208-219. doi: 10.1016/j.atherosclerosis.2014.09.001.

6. Katsiki N, Manes C. Is there a role for supplemented antioxidants in the prevention of atherosclerosis?Clin Nutr. 2009;28:3-9. doi: 10.1016/j.clnu.2008.10.011.

7. Madamanchi NR, Vendrov A, Runge M. Oxidative stress and vascular disease. Arteriosclerosis, thrombosis, and vascular biology. 2005;25:29-38. doi: 10.1161/01. ATV.0000150649.39934.13.

8. Salvayre R, Negre-Salvayre A, Camaré C. Oxidative theory of atherosclerosis and antioxidants. Biochimie. 2016;125:281-96. doi: 10.1016/j.biochi.2015.12.014.

9. Vogiatzi G, Tousoulis D, Stefanadis C. The role of oxidative stress in atherosclerosis. Hellenic J Cardiol. 2009;50(5):4029.

10. Pandit A. Pandey AK. Atherosclerosis: Current perspectives. Apollo Medicine. 2016;13(1):10-16. doi: $10.1016 / \mathrm{j}$.apme.2015.04.002.

11. Aluganti Narasimhulu C, Fernandez-Ruiz I, Selvarajan K, Jiang X, Sengupta B, Riad A, Parthasarathy S. Atherosclerosis--do we know enough already to prevent it? Curr Opin Pharmacol. 2016;27:92-102. doi: 10.1016/j. coph.2016.02.006.

12. Rajendran P, Nandakumar N, Rengarajan T, Palaniswami R, Gnanadhas EN, Lakshminarasaiah U, Gopas J, Nishigaki I. Antioxidants and human diseases. Clinica chimica acta. 2014;436: 332-347. doi:10.1016/j.cca.2014.06.004.

13. Siti HN, Kamisah Y, Kamsiah J. The role of oxidative stress, antioxidants and vascular inflammation in cardiovascular disease (a review). Vascul Pharmacol. 2015;71:40-56. doi: 10.1016/j.vph.2015.03.005.

14. Mitra S, Deshmukh A, Sachdeva R, Lu J, Mehta JL Oxidized low-density lipoprotein and atherosclerosis implications in antioxidant therapy. Am J Med Sci. 2011;342:135-42. doi: 10.1097/MAJ.0b013e318224a147.

15. Gupta RK, Patel AK, Shah N, Chaudhary AK, Jha UK, Yadav UC, Gupta PK, Pakuwal U. Oxidative stress and antioxidants in disease and cancer: a review. Asian Pac J Cancer Prev. 2014;15:4405-9.

16. Mathur P, Ding Z, Saldeen T, Mehta JL. Tocopherols in the prevention and treatment of atherosclerosis and related cardiovascular disease. Clin Cardiol. 2015;38:570-6. doi: $10.1002 /$ clc. 22422 .

17. Husain K, Hernandez W, Ansari RA, Ferder L. Inflammation, oxidative stress and renin angiotensin system in atherosclerosis. World J Biol Chem. 2015;6(3):209-17. doi: $\quad 10.4331 /$ wjbc.v6.i3.209. 
18. Rafieian-Kopaei M, Setorki M, Doudi M, Baradaran A, Nasri H. Atherosclerosis: process, indicators, risk factors and new hopes. Int J Prev Med. 2014;5:927-46.

19. Khan R, Spagnoli V, Tardif JC, L'Allier PL. Novel antiinflammatory therapies for the treatment of atherosclerosis. Atherosclerosis. 2015;240:497-509. doi: 10.1016/j. atherosclerosis.2015.04.783.
20. Nikolic D, Rizzo M, Mikhailidis DP, Wong NC, Banach M. An evaluation of RVX-208 for the treatment of atherosclerosis. Expert Opin Investig Drugs. 2015;24:138998. doi: 10.1517/13543784.2015.1083010.

21. Bruikman CS, Stoekenbroek RM, Hovingh GK Kastelein JP. New Drugs for Atherosclerosis. Can J Cardiol. 2017;33:350357. doi: 10.1016/j.cjca.2016.09.010.

Copyright $\odot 2019$ The Author(s); Published by Society of Diabetic Nephropathy Prevention. This is an open-access article distributed under the terms of the Creative Commons Attribution License (http://creativecommons.org/licenses/by/4.0), which permits unrestricted use, distribution, and reproduction in any medium, provided the original work is properly cited. 